\title{
Monte Carlo Modelling for Domestic Car Use Patterns in United Kingdom
}

\author{
Sikai Huang \\ Department of Electronic and Electrical Engineering \\ University of Strathclyde \\ Glasgow, United Kingdom \\ sikai.huang@strath.ac.uk
}

\author{
David Infield \\ Department of Electronic and Electrical Engineering \\ University of Strathclyde \\ Glasgow, United Kingdom \\ david.infield@strath.ac.uk
}

\begin{abstract}
For the purposes of quantifying the potential impact of widespread electric vehicles charging on the UK's power distribution system, it is essential to obtain relevant statistical data on domestic vehicle usage. Since electric vehicle ownership is presently very limited, these data will inevitably be for conventional internal combustion engine vehicles, and in particular privately owned vehicles. This should not be an issue since the limited journey distances that will dealt with in this work could as easily be undertaken by an electric vehicle as a conventional vehicle. Particular attention is paid to the United Kingdom 2000 Time Use Survey as it contains detailed and valuable statistical information about household car use. This database has been analyzed to obtain detailed car use statistics, such as departure and arrival time, individual journey time, etc. This statistical information is then used to build up two Monte Carlo simulation models in order to reproduce weekday car driving patterns based on these probability distributions. The Monte Carlo methodology is a well-known technique for solving uncertainty problems. In this paper, key statistics of domestic car use are presented together with two different Monte Carlo simulation approaches the simulation results that have been analyzed to verify the results being consistent with the statistics extracted from the TUS data.
\end{abstract}

Keywords-vehicle data; electric vehicle; Monte Carlo modelling;

\section{INTRODUCTION}

There are quite few studies with good quality analysis utilizing household car use data sources in order to capture the characteristics of privately owned or domestic car driving patterns. In papers [1] and [2], the author simulates the plug-in time of individual electric vehicle (EV) by analyzing US National Household Travel Survey (NHTS) 2001 data, [3]. NHTS data are collected on daily trips taken in a 24-hour period, and various attributes are recorded, such as purpose of the trip, means of transportation used, and how long the trip took, etc. In [4] and [5], driving patterns were extracted from the transportation data for the year 2008 provided by the Dutch Ministry of Transportation, [6]. The Mobility Research Netherlands report gives a large dataset of individual trips by various transport means. For the purposes of quantifying the potential impact of widespread electric vehicles charging on the UK's power distribution system, it is essential to obtain relevant statistical data on domestic vehicle usage. Since EV ownership is presently very limited, this data will inevitably be for conventional internal combust engine (ICE) cars, and in particular privately owned ones.

In this paper, key statistics of UK domestic car use are presented in Section II. Section III illustrates two Monte Carlo (MC) models that have been structured with two contrasting simulation approaches to generate random samples from statistics obtained from the survey data so that synthesized daily car use can be simulated. In Section IV, simulation results of both MC models will be analyzed and compared with input statistics. The strengths and weakness of the two approaches will be discussed in the Section V.

\section{Key Statistics OF DOMESTIC CAR Use}

\section{A. Available Domestic Vehicle Data Sources}

In order to build up the probabilistic models for privately owned car use, it is important to identify appropriate vehicle travel database. The United Kingdom 2000 Time Use Survey (TUS) has been selected as the main data source because of its high, ten-minute time resolution, [7]. The TUS data focus on household daily activities, which including driving. There are other data sources available for verifying key statistics, such as time of day of car departure and arrival home, average daily driving journey time and length, car parking locations, and the journey purpose; in particular, the National Travel Survey 20022010 (NTS), and Focus on Personal Travel 2005, [8] and [9]. These surveys are based on diaries kept by thousands of participants describing their day-to-day activities. In particular, the TUS data provide detailed data on privately owned vehicle use. Individuals in sampled households are asked to complete travel diaries for each week during survey period. Each diary runs over a 24-hour period. These diaries provide details of trips undertaken, including the purpose, method of travel, time of day that journey takes place, and the destination.

The main advantage of using TUS as the main data source is the higher time resolution compared to the NTS data, despite the rather similar approaches to collecting data, and the nature of the records. Thirty-minute or lower time resolution data certainly lose critical information on commonly made short journeys, such as trips to local shops. The amount of energy required to charge electric vehicle batteries is calculated based on the total length of journeys starting from and returning back home. As a result, the amount of energy required to charge EV batteries for 


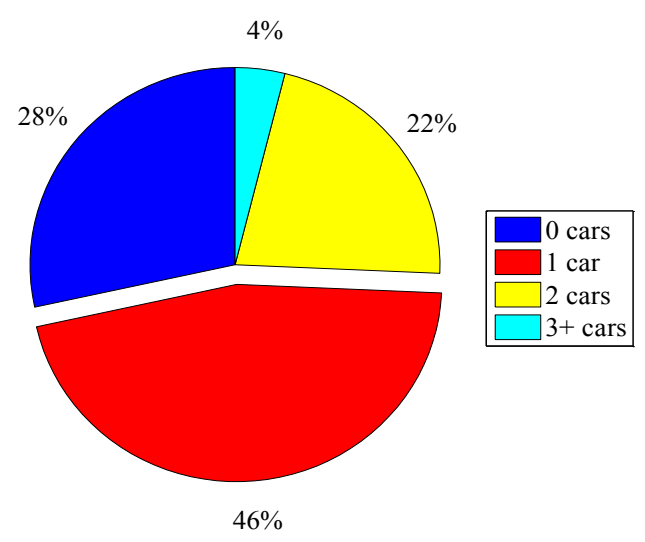

Fig. 1. Cars ownership per household.

these frequent short journeys could be neglected with lower time resolution data.

\section{B. Methods Used for Calculating Vehicle Use Statistics}

Different stages of data processing have been undertaken to obtain household driving statistics. The first step is raw data processing, which extracts and converts the original TUS data into more usable data compatible with Microsoft Excel. The next step is data post processing, and the outcome dataset contains household diaries together with car ownership information. These processed datasets have been further analyzed in order to obtain statistics for the use of privately owned cars in a form useful for the subsequent MC modelling.

\section{Identification of Key Statistics in Time of Use Survey}

The number of cars per household is analyzed as shown in Fig. 1. The largest group of households in the survey have just one car. Just over one-fourth of total households have two cars. 28 percent of households in the TUS database do not have a car. The average car ownership per household is just over one for the entire sample population.

The time of day when people use their cars as the main driver (i.e. not as passenger) is important as it determines when EV batteries are being discharged and by how much, and consequently will affect when battery charging is likely to occur. Note that as defined in the survey data, a car is driving on the road at a particular time of day when the diary gives the household adult activity for that ten-minute period as "driving a car as main driver" (code 15 in TUS). The exact timing of battery charging is essential to determine the added load on the electricity distribution system, in itself important to ensure the provision of adequate electricity supply capability (both generation, transmission and local distribution) to charge future electric vehicles whilst supplying the remainder of the demand. The probabilities of a car being in use for weekdays and weekends are calculated from TUS data and NTS data as shown in Fig. 2.

The varying probability through a weekday that a random car is being driven exhibits the expected peaks in the morning and evening. In contrast, driving over the weekend is more widely spread over the day. Specifically the results confirm that the peak driving time for weekdays is over the period 7:30 to $9: 20$ in the morning and $16: 45$ to $18: 40$ in the evening as might be

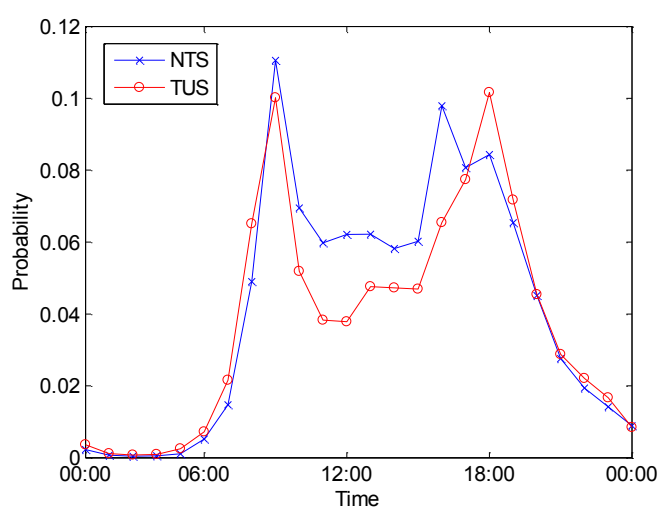

(a)

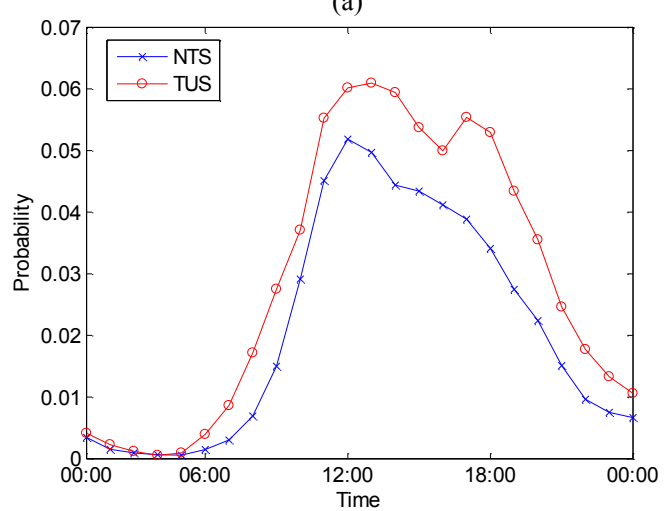

(b)

Fig. 2. Comparison of car in use. (a) weekday; (b) weekend.

expected from known commuting behavior, and that the pattern over the weekend is very different, justifying the disaggregation of the data.

The amount of time people spend on actual driving is important for calculating the amount of energy used by the EV, the state of charge, and the re-charging period required. Across the entire fleet of EVs it will determine the amount of electricity required to replace the equivalent number of conventional ICE cars. Up-to-date EV specifications indicate that it is not possible to completely replace ICE cars due to range limitations. By counting the number of individual driving periods in the weekday diaries, a probability distribution of the length of individual journeys can be deduced. Home is regarded as the primary location for EV charging. Attention is now turned to the aggregate or cumulative time driving during any period away from home. For a given value of time $t$, this is found simply by summing up all the individual journey durations within the time period between a departure and the next arrival, and then calculating the probabilities for each of the durations so as to give the probability distribution of aggregate driving duration for journeys at arrival time $t_{\text {arrival }}$ as illustrated in Fig. 3. A bird's eye view of the conditional probability of driving period given the time of arrival at home, shows that at the beginning of the diary day (4am) and its end (3:50am next day), the probability of certain driving times is equal to 1 . This is the result of the very small sample sizes at such times of day since very few cars arriving home then. For example, for a car arriving home at 4:10am, there is only one diary record, so that the calculated probability is unity for the one round trip journey involved, in 


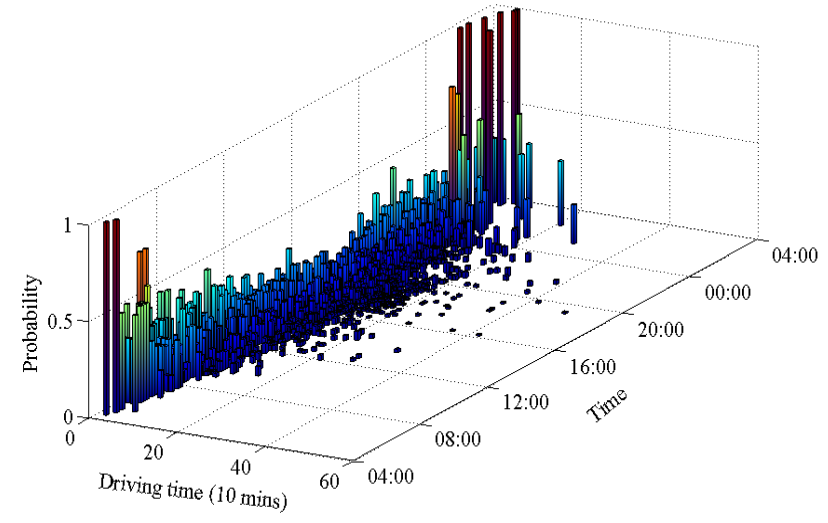

Fig. 3. Probability distributions that cumulative car driving period dependent on the arrival home time.

this particular case it was equal to twenty minutes. Similar arguments apply to other small samples sizes, giving probabilities of $0.5,1 / 3,0.25$, etc.

Driving implies that the car is away from home. Therefore, the total time driving also depends on the period that the car is away from home, and clearly is less than or equal to the away period. In the previous context, the total amount of time spent on the driving has been calculated when car arrives home. Since there is a one to one relationship between the car arrival times and the car departure times, the same one to one relationship between car away period and total time driving will result when car departs from home.

\section{Monte CARlo Simulation of Domestic CAR DRIVING PATTERNS}

Monte Carlo (MC) simulation is widely regarded as the best approach to generate synthetic individual car use patterns. The basic concept of the Monte Carlo method dates back to the 18th century when the French scientist Buffon presented the famous needle throw test method to calculate $\pi$ in 1733, [10] and [11]. In previous section, probability distributions of weekday car daily use have been calculated from the TUS data. Now the task is to reproduce weekday car driving patterns based on these probability distributions. In order to achieve this, MC models have been structured to generate random samples directly from these probability distributions so that synthesized daily car use can be simulated. This sampling approach takes advantage of the high time-resolution of the TUS data as well as significantly reduce the mathematical complexity, such as curve fitting and copula techniques, presented in [12] and [4]. Two modelling approaches for structuring the MC simulation have been implemented. In this research, the $\mathrm{MC}$ model generates random samples directly from probability distributions taken from the TUS data rather than from fitted distribution functions.

\section{A. Inverse-Transform Method for Discrete Distribution}

The basic idea of this transformation method is to generate random samples from the given target distribution using the cumulative distribution function (CDF), by using random numbers created over the interval, $U \in[0,1]$, with uniform probability distribution. This approach to generating random samples for MC simulation is known as the Inverse-Transform
Method (see [13] and [14]). The inverse-transform algorithm can be implemented as follows:

1. Generate random number $U \sim U \in[0,1]$.

2. Find the smallest positive integer, $k$, such that $U \leq$ $\mathrm{F}\left(x_{k}\right)$ and return the sample from random variable (RV) $X$ as $x_{k}$

Note that in general, the most time consuming process is Step 2, which makes comparisons between the random number and the given by the CDF of $X$. For some RVs that will be used in the MC modelling described in the following section, there are only two possible states and in this case the inversetransform method is computationally fast. It simply comprises of a check whether the random number $U$ is less than or greater of equal than the probability of state 1 . The inverse-transform method is an effective tool for generating RVs sampled from a specified distribution. Their implementation requires a reliable random number generator; these are readily available in most program environments, such as the MATLAB function 'rand' that generates uniformly distributed random numbers between 0 and 1. Besides inverse-transform method, Markov Chain method has also been considered by utilizing car transient status probabilities, such as in [15]. Nevertheless, the inversetransform method takes the advantage of the high resolution of TUS data by directly sampling from these car use probability distributions.

\section{B. Multiple Car Locations Monte Carlo Modelling Approach}

This approach follows the car location and thus the daily car use patterns in relation to home can be generated. The model determines the car's initial location first by sampling from the two state probability distribution that car parking at home, $P_{\text {park }}$. Depending on the initial location, the model then generates car departure and arrival samples from two conditional two state probability density functions (pdf), $P_{\text {departure }}$ and $P_{\text {arrival }}$. This model only uses $P_{\text {departure }}$ and $P_{\text {arrival }}$ for initialization, after which the loop generating car away period, $T_{\text {away }}$ and car parking period, $T_{\text {park }}$ is repeated as 'hopping process' from the conditional pdfs, car away period dependent on departure time, $P\left(T_{\text {away }} \mid t_{\text {departure }}\right)$ and car parking period dependent on arrival time, $P\left(T_{\text {park }} \mid t_{\text {arrival }}\right)$. Fig. 4 shows the overview of this approach MC modelling. The final part of this model generates samples for cumulative car driving period dependent on car away period from the conditional pdf, $P\left(T_{\text {driving }} \mid T_{\text {away }}\right)$. This approach can be regarded as a next-event time-advance mechanism in which time can progress in multiples of the tenminute time step determined by sampling from a cumulative distribution function (cdf) of duration (for example of car parking at home). Therefore, this model is named as multiple time increments Monte Carlo model.

\section{Car Return Home Monte Carlo Modelling Approach}

The car return home approach focuses on household activity related to car use, and generates random samples for two discrete $\mathrm{RVs}$. The model is named as return time dependent MC model. The first is sampled from the time dependent two state $p d f$ $P_{\text {arrival household }}$ for a car arriving back home at a specific household. The second is sampled from the multi-state $p d f$, 


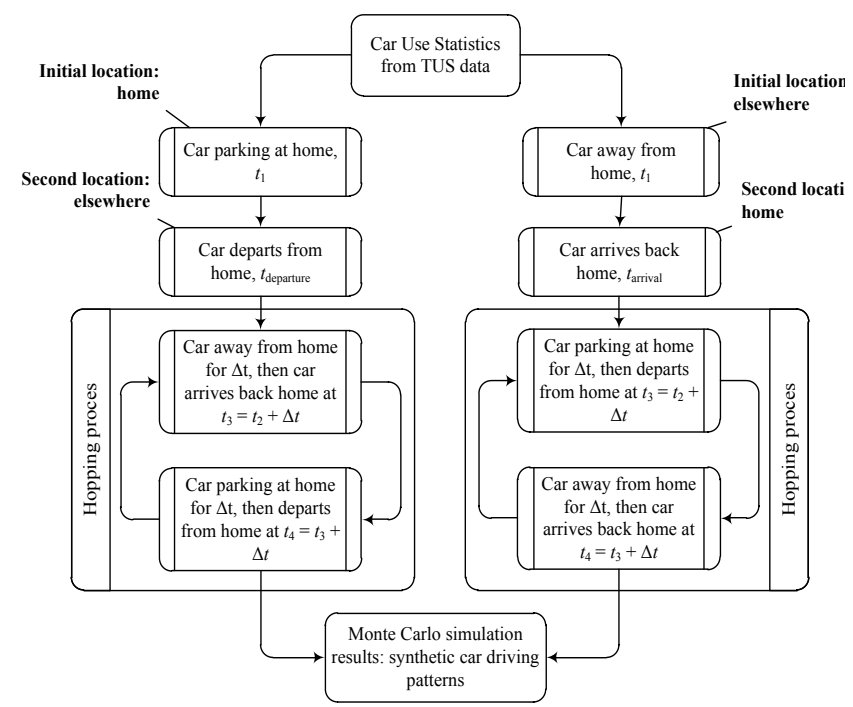

Fig. 4. Overview of the conventional approach to MC modelling.

$P\left(T_{\text {driving }} \mid t_{\text {arrival }}\right)$ for cumulative car driving period dependent on car arrival time at the household. The model thus contains two sub-models as shown in Fig. 5; the first one generates random samples for the two state RVs representing a car arriving back home, denoted $p_{\text {arrival_household }}$ and a second that generates random samples for the RV representing cumulative driving time prior to arrival home, denoted $p_{\text {driving }}^{t_{\text {arrival }}}$.

\section{Analysis of Monte Carlo Simulation Results}

To ensure that both Monte Carlo models do not significantly distort the simulation results, a careful statistical analysis of the results has been performed, comparing the driving patterns simulated with those of the original TUS data, [16]. Before this can be done, it is essential to ensure convergence of the Monte Carlo calculations. A statistical consistency analysis can then be done to determine whether or not the MC simulation results reflect the original input data.

\section{A. Diagnosing Convergence of Simulation Results}

A more sophisticated method to assess convergence is the acceptable shifting convergence band rule, [17]. This method defines that the simulation has converged when the sample mean of the output variable of interest falls inside the convergence band (CB) of a given width and length; otherwise, the convergence band shifts to a new sample mean outside the existing $\mathrm{CB}$ with a new width and length. The convergence of MC simulation depends on the number of trials or iterations performed. For the car modelling, convergence can be taken as convergence of the mean driving period, $E\left[X_{\text {driving }}\right]$. For the purpose of identifying convergence of both MC models, iterations have been performed as shown in Table I.

TABLE I. NUMBER OF ITERATIONS FOR MC MODELS

\begin{tabular}{|l|l|l|}
\hline \multicolumn{1}{|c|}{ MC Modelling } & Iterations & Days \\
\hline Simplified approach & 10,000 & 1 \\
\hline Conventional approach & 20,000 & 5 \\
\hline
\end{tabular}

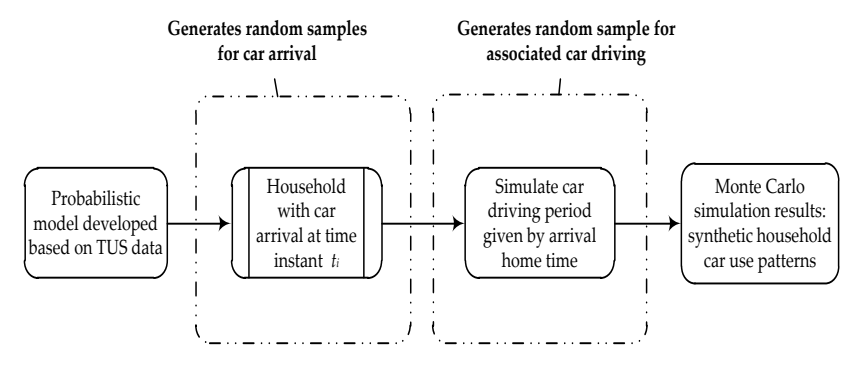

Fig. 5. The structure of simplified approach Monte Carlo modelling.

\section{1) Multiple Time Increments MC model}

After applying the convergence criteria, the mean value converged at $i=9930$ with the $90 \%$ confidence interval and at $i$ $=9795$ with the $95 \%$ confidence interval. The resulting the mean value equals to 5.3083 and 5.3071 for confidence level of $90 \%$ and $95 \%$ respectively. For practical purposes the conventional $\mathrm{MC}$ model estimates that a typical car is driving for $5.3 \%$ of the time as shown in Fig. 6a.

\section{2) Return Time Dependent MC Model}

Applying the convergence criteria described in section IV- $A$, the mean value is deemed to have converged by $i=9051$ with a $90 \%$ confidence interval and $i=8192$ with the $95 \%$ confidence interval. It is clear that sufficient iterations have been undertaken; the converged values are equal to 4.7427 and 4.7336 for confidence levels of $90 \%$ and $95 \%$ respectively. As a result, the simplified MC model estimates that a typical car is driving for $4.7 \%$ of the time as shown in Fig. $6 \mathrm{~b}$.

\section{B. Consistency Analysis of Monte Carlo Modelling Results}

Direct comparison approach in which the consistency of the MC simulation results with TUS data has been established,

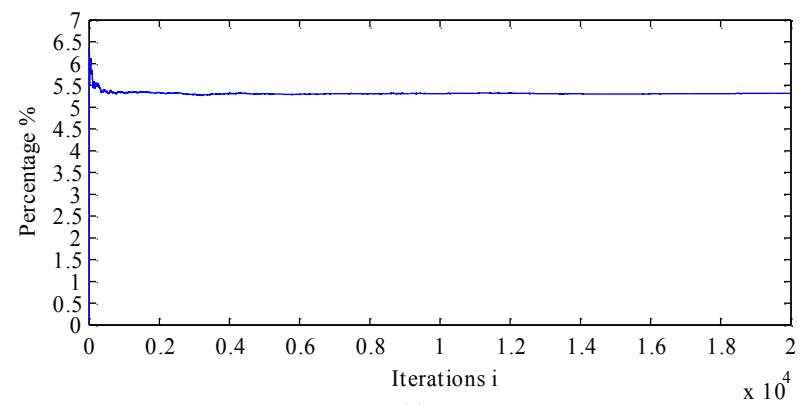

(a)

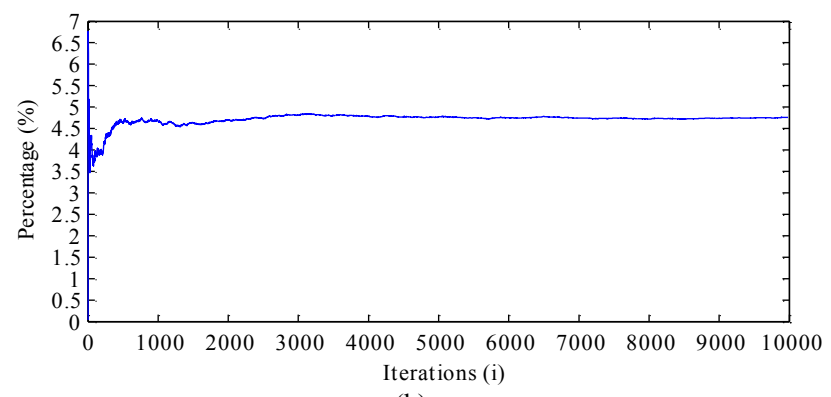

(b)

Fig. 6. Convergence of mean driving period over 24 hour as percentage. (a) multiple time increments MC model; (b) return time dependent MC model 


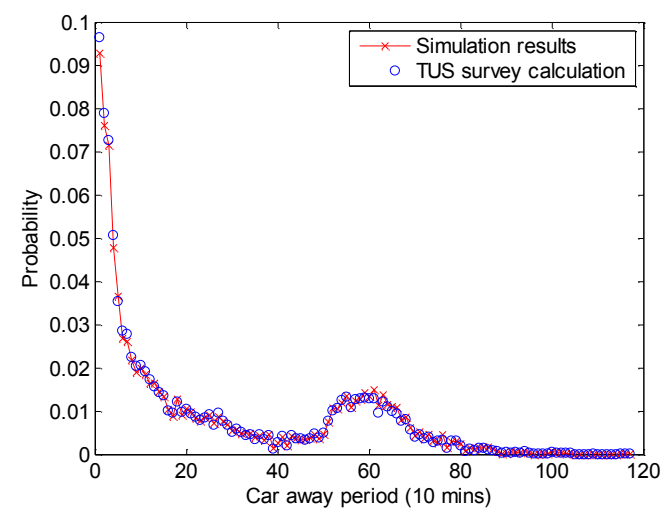

(a)

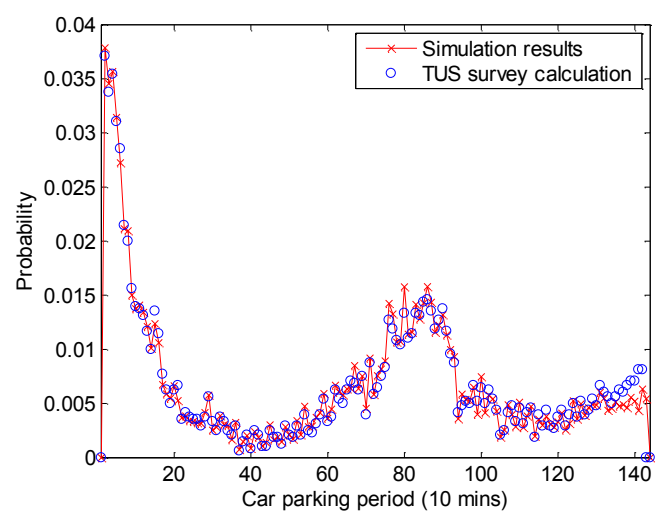

(b)

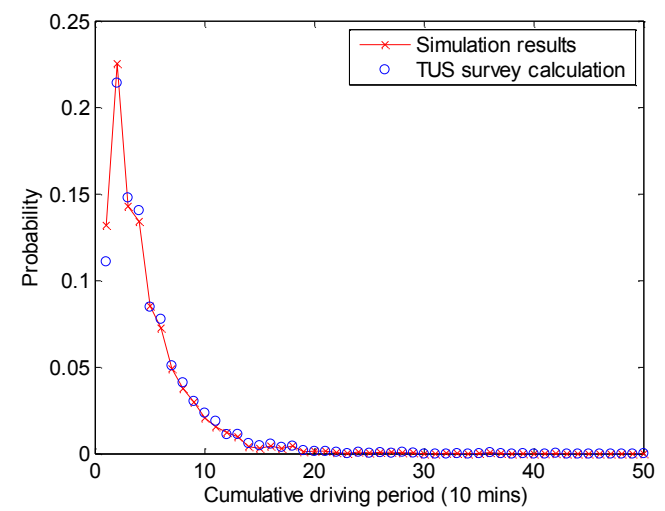

(c)

Fig. 7. Comparison of TUS data and MC simulation results. (a) car away period, (b) car parking period, (c) car driving period.

where TUS data are directly used to create the Monte Carlo model, the model output can be directly compared to the input. In this case, percentage error has been calculated between MC model results and TUS data in order to check the consistency.

\section{1) Multiple Time Increments MC Model}

The multiple time increments MC model uses more car use statistics than the car return home approach. The car away period is directly sampled from the relevant probability distribution, as is the car parking period. The probability distributions of car away period and car parking period have been compared. Fig. $7 \mathrm{a}$ and $\mathrm{b}$ show the comparison, and it is clear that there is a good correlation. The cumulative car driving period is sampled as conditional on car away period. The probability distribution of

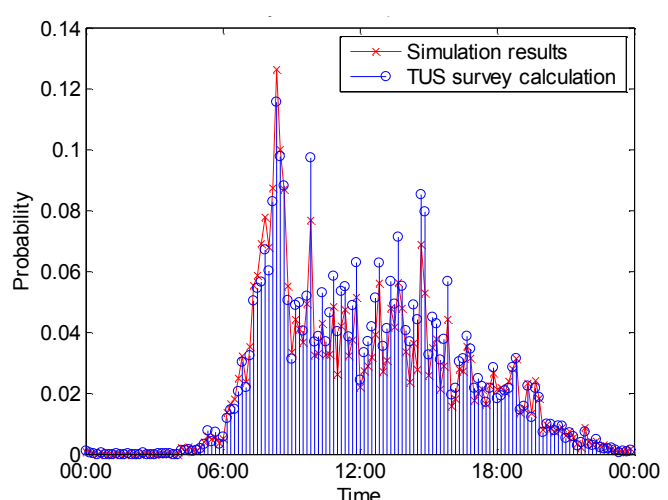

(a)

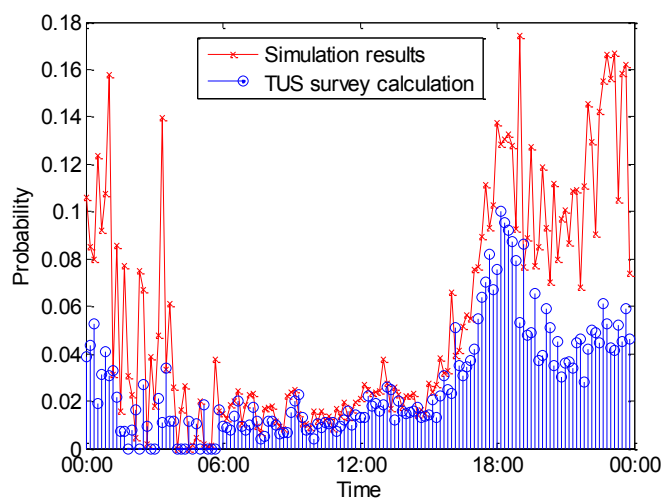

(b)

Fig. 8. Comparison of multiple time increments MC results and TUS calculation. (a) Probability of car departure; (b) Probability of car arrival.

cumulative car driving period provides good correlation with the TUS data as shown in Fig. 7c. The consistency check has also been performed for the statistics that indirect used in this $\mathrm{MC}$ model, such as car departure and car arrival. Good correlation has been illustrated for car departure probabilities as shown in Fig. 8a. The percentage error for probability of a car departure is only $1.4 \%$. However, the probability of car arrival home is poorly modelled as shown in Fig. 8b. Due to the complexity of this particular MC model, a further analysis of these simulation results need to be undertaken and it is considered as future work.

\section{2) Return Time Dependent MC Model}

A key outcome of return time dependent MC model, the probability of household car arrival time, has been validated against probabilities calculated directly from the TUS weekday dataset. Fig. 9 shows the good correlation for the probability that a car arrives back at the household based on 10,000 MC simulations with those based on the original TUS data. Fig. 10 shows the comparison for cumulative car driving period dependent on the time when car arrives back home. As expected, the return time dependent MC model reproduces accurate car driving period statistics. The percentage of difference has been calculated for both probability of a car returns to house and cumulative driving period dependent on car arrival time. The percentage error of probability of a car returns to house is $0.37 \%$, and for cumulative driving, the percentage error is less than $1 \%$. Therefore, the return time dependent MC model produces acceptably accurate results. 


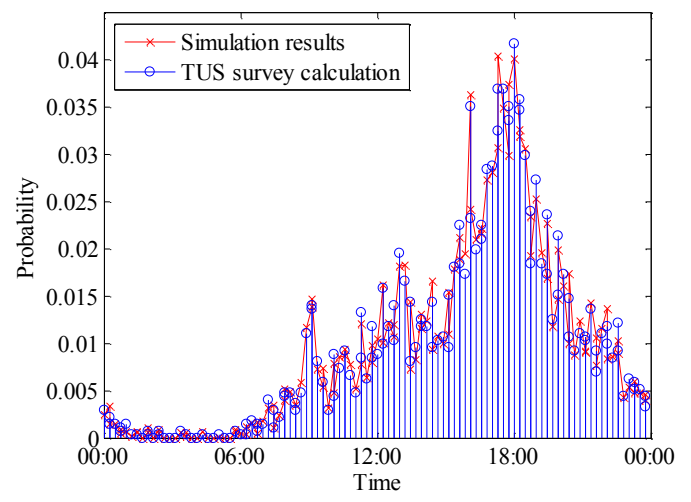

Fig. 9. Comparison of probability that a car returns to house.

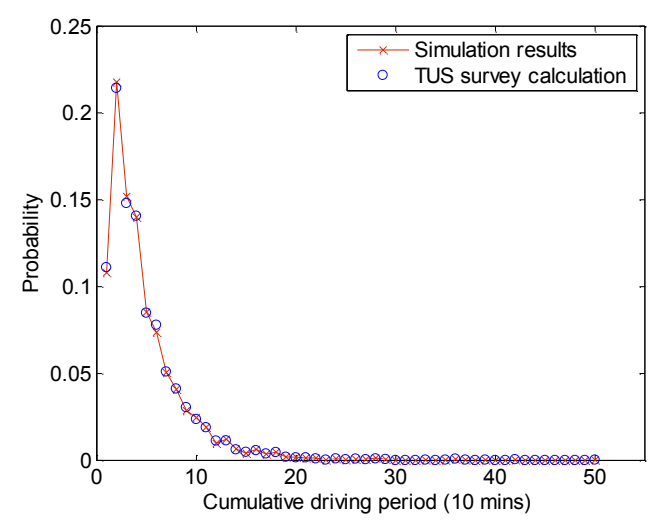

Fig. 10. Comparison of cumulative car driving period.

\section{CONCLUSION}

In this paper, key statistics of UK household car use has been presented. The reasons for choosing United Kingdom Time of Use Survey 2000 data as the main data source for this research was explained, in particular its much higher time resolution than other UK sources such as the National Travel Survey. In fact the time resolution, at ten minutes, is better than datasets available to most researchers around the world, where hourly data are the most commonly used. Detail analysis of privately owned car use in the UK shows that usage behavior has significant differences between weekdays and weekends. Preliminary analysis shows that privately owned cars are utilized only $5.2 \%$ of the time for transportation, thus making them, in principal, available for the remaining $94.8 \%$ of time providing ancillary services to the power system. The focus of this research is on car use during the working week as this is where most power network problems are anticipated. Probabilistic characterization of car usage during weekdays has been used as the basis for MC simulations of car use. Two different approaches of Monte Carlo modelling have been described in detail. The advantage of the car return home approach is that it utilizes only two car use statistics, which enables MC simulation results to converge with relatively less iterations. Therefore, less computational time is required compared to the multiple car locations approach considered. The disadvantage of the car return home approach is that only part of the car use patterns can be captured; nevertheless it should give a reasonable estimate and time distribution of car charging, which is the main objective of the MC models. The multiple car location approach can generate weekday multiple car parking locations in relation to home. It provide information about the next car departure, which could be helpful in the study of demand side management applied to EV charging. Good correlation has been shown for the statistics directly been used in the model. However, poor agreement occurs for car arrival events. Further analysis need to be performed in order to explained this particular problem, and it is considered as part of the future research.

\section{ACKNOWLEDGMENT}

This work was supported by the UK's Engineering and Physical Sciences Research Council (EPSRC) under Realizing Transition Pathways project EP/K005316/1.

\section{REFERENCES}

[1] Shengnan Shao; Pipattanasomporn, M.; Rahman, S., "Grid Integration of Electric Vehicles and Demand Response With Customer Choice," Smart Grid, IEEE Transactions on , vol.3, no.1, pp.543,550, March 2012.

[2] Taylor, J.; Maitra, A.; Alexander, M.; Brooks, D.; Duvall, M., "Evaluation of the impact of plug-in electric vehicle loading on distribution system operations," Power \& Energy Society General Meeting, 2009. PES '09. IEEE, vol., no., pp.1,6, 26-30 July 2009.

[3] National Household Travel Survey Oakridge National Laborotary, 2001 [Online]. Available: http://nhts.ornl.gov/index.shtml

[4] Lojowska, A.; Kurowicka, D.; Papaefthymiou, G.; Van der Sluis, L., "Stochastic Modeling of Power Demand Due to EVs Using Copula," Power Systems, IEEE Transactions on , vol.27, no.4, pp.1960,1968, Nov. 2012

[5] Verzijlbergh, R.A.; Grond, M.O.W.; Lukszo, Z.; Slootweg, J.G.; Ilic, M.D., "Network Impacts and Cost Savings of Controlled EV Charging," Smart Grid, IEEE Transactions on , vol.3, no.3, pp.1203,1212, Sept. 2012

[6] Ministry of Transport, Public Works and Water Management, "Mobiliteitsonderzoek Nederland (in Dutch)," April 2009, last visited October 2010. [Online]. Available: www.mobiliteitsonderzoeknederland.nl

[7] Ipsos-RSL and office for National Statistics, United Kingdom Time Use Survey, 2000 [computer file]. $3^{\text {rd }}$ Edition. Colchester, Essex: UK Data Archive [distributor], September 2003. SN: 4504.

[8] National Statistics, Department for Transport. 'Transport statistics bulletin-national travel survey: 2008,' $9^{\text {th }}$ April 2009.

[9] Department for Transport, 'Focus on personal travel: 2005 Edition', National Statistics, UK Data Archive Study Number 5340, April 2005.

[10] Weisstein, Eric W."Buffon's Needle Problem." From MathWorld--A Wolfram Web $\quad$ Resource. http://mathworld.wolfram.com/BuffonsNeedleProblem.html

[11] H. Solomon, "BUFFON NEEDLE PROBLEM, EXTENSIONS, AND ESTIMATION OF $\pi$," in Geometric Probability, 1st ed. Philadelphia. City of Publisher: Society for Industrial and Applied Mathematics, 1978, ch. 1, pp. 1-5.

[12] Tae-Kyung Lee; Bareket, Z.; Gordon, T.; Filipi, Z.S., "Stochastic Modeling for Studies of Real-World PHEV Usage: Driving Schedule and Daily Temporal Distributions," Vehicular Technology, IEEE Transactions on , vol.61, no.4, pp.1493,1502, May 2012

[13] R. Y. Rubinstein and D. P. Kroese, Simulation and the Monte Carlo Method: Wiley, 2011.

[14] Whiteley, N. (2010) Monte Carlo Methods. [Lecture Notes] Department of Mathematics, University of Bristol.

[15] F. Soares, J. P. Lopes, P. R. Almeida, C. Moreira, and L. Seca, "A stochastic model to simulate electric vehicles motion and quantify the energy required from the grid," PSCC, Stockholm, Sweden, 2011.

[16] M. Guizani, A. Rayes, B. Khan, and A. Al-Fuqaha, Network Modeling and Simulation: A Practical Perspective: Wiley, 2010.

[17] Mustafa Y. Ata, A convergence criterion for the Monte Carlo estimates, Simulation Modelling Practice and Theory, Volume 15, Issue 3, March 